\title{
Pathways and role of MALAT1 in esophageal and gastric cancer (Review)
}

\author{
ATHANASIOS SYLLAIOS ${ }^{1}$, DIMITRIOS MORIS ${ }^{2}$, GEORGIA SOFIA KARACHALIOU ${ }^{3}$, \\ STRATIGOULA SAKELLARIOU ${ }^{4}$, IOANNIS KARAVOKYROS ${ }^{1}$, MARIA GAZOULI $^{5}$ and DIMITRIOS SCHIZAS ${ }^{1}$
}

${ }^{1}$ First Department of Surgery, National and Kapodistrian University of Athens, Laikon General Hospital, Athens 11527, Greece; ${ }^{2}$ Department of Surgery, Duke University Medical Center; ${ }^{3}$ Department of Medicine, Division of Hematology/ Oncology, Duke University Medical Center, Durham, NC 27707, USA; ${ }^{4}$ First Department of Pathology and ${ }^{5}$ Laboratory of Biology, Medical School, National and Kapodistrian University of Athens, Athens 11527, Greece

Received September 24, 2020; Accepted January 5, 2021

DOI: $10.3892 / \mathrm{ol} .2021 .12604$

\begin{abstract}
Esophageal cancer (EC) and gastric cancer (GC) often have an unfavorable prognosis. Therefore, research is being conducted to identify the molecular mechanisms underlying the tumorigenesis and progression of GC and EC, and to indicate novel therapeutic targets and clinically applicable biomarkers. The dysregulations and roles of long non-coding RNAs (lncRNAs) have been widely reported, and current published literature has shown that IncRNAs play important regulatory roles in the carcinogenesis and progression of EC and GC. The lncRNA metastasis-associated lung adenocarcinoma transcript 1 (MALAT1) has been investigated in a number of studies with regard to its pathogenic pathways and association with the prognosis of gastric and esophageal malignancies. As literature on the topic of MALAT1 in EC and GC continues to emerge, the present review aims to summarize all current knowledge on the association between MALAT1 expression and esophagogastric malignancies and to describe the pathogenic pathways and possible prognostic role of MALAT1 in esophagogastric cancer. As research studies on MALAT1 pathways in esophagogastric malignancies are ongoing, new possibilities for the diagnosis, prognosis and therapy of GC and EC are likely to be identified.
\end{abstract}

\section{Contents}

1. Introduction

2. MALAT1 and esophageal cancer

3. MALAT1 and gastric cancer

4. Conclusions

Correspondence to: Dr Athanasios Syllaios, First Department of Surgery, National and Kapodistrian University of Athens, Laikon General Hospital, 17 Agiou Thoma Street, Athens 11527, Greece

E-mail:nh_reas@hotmail.com

Key words: MALAT1, biomarker, long non-coding RNA, esophageal cancer, gastric cancer

\section{Introduction}

Esophageal cancer (EC) and gastric cancer (GC) are leading causes of cancer-associated mortality. Despite recent advances in surgical treatment, chemotherapy, radiotherapy and molecular-targeted treatment, the five-year survival rate of patients with GC or EC remains poor (1). Further research is therefore required to elucidate the molecular mechanisms underlying the tumorigenesis and progression of GC and EC and to identify novel therapeutic targets. Also, applicable biomarkers for predicting clinical outcome are imperatively required.

Studies identifying potential biomarkers of tumorigenesis have routinely focused on protein-coding genes. However, it has been estimated that $98 \%$ of the human genome is non-protein coding. Ground-breaking discoveries in the field of non-coding RNAs have helped to improve the characterization of microRNAs and long non-coding RNAs (lncRNAs) (2). IncRNAs are transcribed RNA molecules that are $>200$ nucleotides in length and located in nuclear or cytosolic fractions, yet do not encode proteins $(3,4)$. The abnormal expression and roles of lncRNAs in cancer have been reported in numerous studies. IncRNAs may play important roles in various biological processes, including immune responses, differentiation, angiogenesis, apoptosis, cell proliferation and autophagy. Therefore, they may contribute to the prognosis and treatment of cancer as novel therapeutic targets (5).

Metastasis-associated lung adenocarcinoma transcript 1 (MALAT1) is a lncRNA that has been revealed to be dysregulated and have prognostic and therapeutic significance in various cancers, including lung cancer, pancreatic cancer, hepatocellular carcinoma, nasopharyngeal carcinoma, osteosarcoma, colorectal cancer, breast cancer, GC and EC (3). A number of studies have investigated the pathogenetic pathways of MALAT1, and the association between MALAT1 expression and the prognosis of gastric and esophageal malignancies. The present review aims to summarize all current knowledge on the association between MALAT1 expression and these malignancies, and to describe the pathogenetic pathways and the possible prognostic role of MALAT1 in esophagogastric cancer. 


\section{MALAT1 and esophageal cancer}

MALAT1 pathways in esophageal squamous cell carcinoma $(E S C C)$. In patients with ESCC, MALAT-1 is expressed at higher levels in cancer tissues compared with paired adjacent normal tissues, and in ESCC cell lines, MALAT-1 knockdown reduces proliferation, increases apoptosis, inhibits migration, invasion and colony formation, and leads to cell cycle arrest at the G2/M phase (6). MALAT1 depletion has been shown to induce $\mathrm{G} 2 / \mathrm{M}$ phase arrest via phosphorylation of the ataxia-telangiectasia mutated/checkpoint kinase 2 pathway and increase the percentage of apoptotic cells (7). IncRNAs are able interact with miRNAs (miRs), modulate the expression of other lncRNAs, and serve as competing endogenous RNAs (ceRNAs) to miRNAs. Moreover, the expression of lncRNAs can be inhibited by miRNAs through an argonaute 2-mediated pathway. miR-101 and miR-217 have been demonstrated to act as tumor suppressor genes by targeting MALAT1 during ESCC development (8). Furthermore, miR-101 and miR-217 have been found to be downregulated in EC. There is evidence to suggest that the posttranscriptional silencing of MALAT1 by miR-101 and miR-217 occurs in ESCC cells, which may significantly suppress the proliferation of the cells via G2/M cell cycle arrest. This may be due to suppression of the MALAT1-mediated upregulation of p21 and p27 expression and inhibition of B-MYB expression. In addition, the overexpression of miR-101 or miR-217, or transfection with MALAT1 small interfering RNA (siRNA) inhibits the migration and invasion of ESCC cells, which may be due to the dysregulation of genes downstream of MALAT1, such as MIA SH3 domain ER export factor 2, hepatocyte nuclear factor $4 \gamma$, roundabout guidance receptor 1, chaperonin containing TCP1 subunit 4 and collagen triple helix repeat containing 1 (8).

miR-101-3p has been demonstrated to bind directly to MALAT1. This miRNA blocks the MALAT-1-induced activation of the PI3K/AKT signaling pathway, resulting in repression of the proliferation, migration and invasion of ESCC cells (2). Furthermore, with the introduction of miRNA-based cancer therapy, Li et al (9) designed a poly(glycidyl methacrylate) (PGEA)-based star-like polycation with flanking folic acid (FA) ligands (S-PGEA-FA), rich in hydrophilic hydroxyl and secondary amine groups, as an effective nanovector with the ability to deliver miR-101 and miR-217 and thereby silence MALAT1 in ESCC cells. The authors found that miR-101 and miR-217 acted like silent information regulators-M to inhibit ESCC cells via the downregulation of MALAT1. S-PGEA-FA was shown to transfer miR-101 and miR-217 into ESCC cells and thereby induce cell cycle arrest, and inhibit cell proliferation, migration and invasion, indicating that it has potential as a promising agent in ESCC therapy (9).

Enhancer of zeste homolog 2 (EZH2) has been shown to be associated with carcinogenesis and cancer progression, and to serve a crucial role in the apoptosis, invasion and migration of cancer cells. MALAT1 is able to promote the malignant development of ESCC by targeting EZH2. Chen et al (10) found that MALAT1 affects the epithelial-to-mesenchymal transition (EMT) and metastasis of EC cells via the EZH2-Notch1 signaling pathway. Another study indicated that MALAT1 may serve as a ceRNA controlling the expression of ZEB1 and ZEB2 via the sponging of miR-200a, and thereby promote the invasion and migration of EC cells through the induction of EMT (11). Furthermore, it has been shown that the silencing of MALAT1 downregulates the expression of OCT4 and NANOG genes, which inhibits EC cell proliferation, migration and tumor sphere formation, while increasing cell apoptosis. The expression of $\beta$-catenin, Lin28 and EZH2 genes is also decreased when MALAT1 is downregulated, while the overexpression of EZH2 completely reverses the MALAT1 siRNA-mediated repression of $\beta$-catenin and Lin28 in EC cells (12).

MALAT1 has been indicated to be associated with the stemness of ESCC cells, as the knockdown of MALAT1 decreases cancer stem cell-like traits. The expression levels of the stemness markers sex-determining region Y-box 2 (SOX2) and NANOG in ESCC cells are reduced by MALAT1 downregulation, as is the migration ability of the cells. MALAT1 positively regulates the stemness of ESCC cells. It binds directly to yes-associated protein (YAP), a transcriptional factor that plays a critical role in the expansion of cancer stem cells, and induces YAP protein expression and transcriptional activity (13).

MALAT1 pathways in ESCC are summarized in Table I and presented in Fig. 1.

Impact of MALAT1 expression in patients with ESCC. In a large Chinese study of single nucleotide polymorphisms of MALAT1, the authors proposed that polymorphism rs3200401 $\mathrm{C}>\mathrm{T}$ is associated with a higher risk of ESCC and polymorphism rs619586 A $>\mathrm{G}$ might be protective against ESCC (14).

The upregulation of MALAT1 in ESCC tissues can influence tumor progression and may predict postoperative survival; therefore, the expression level of MALAT1 can be used as an index to predict the prognosis of patients with ESCC. It has been shown that the expression levels of MALAT1 in ESCC tissues are higher than those in adjacent non-carcinoma tissues, and the overall survival (OS) and disease-free survival (DFS) of patients with high MALAT1 expression levels are shorter than those with low expression levels (OS, 32 vs. 57 months; DFS, 27 vs. 54 months, respectively). Moreover, high MALAT1 expression is associated with a high TNM stage (stage III vs. I and II) (15). High MALAT1 expression has also been shown to be significantly associated with lymphatic invasion, large primary tumor size, distant metastasis and poor tumor differentiation, but not with age, sex or tumor location $(7,16)$. In the only published meta-analysis regarding the role of MALAT1 in ESCC prognosis, a significant association was detected between the expression of MALAT1 and the OS of patients with ESCC in China. More specifically, a high MALAT1 expression level in ESCC tissues was found to be an important independent predictive factor of poor OS and DFS (3).

MALAT1 has been indicated to be associated with the chemo- and radiosensitivity of ESCC cells, with the downregulation of MALAT1 enhancing the chemo- and radiosensitivity of ESCC cells $(2,13)$. Cyclin-dependent kinase subunit 1 (Cks1) is frequently overexpressed in ESCC, and its overexpression is associated with the increased resistance of ESCCs to radiotherapy. Li et al (17) found that the expression levels of MALAT1 and Cks1 were reduced in ESCC mouse xenografts and cells when irradiated. Furthermore, the irradiation-induced reduction in cell viability, increase in apoptosis and downregulation of Cks1 were inhibited by 
Table I. Summary of MALAT1 pathways in ESCC.

\begin{tabular}{|c|c|c|c|c|}
\hline First author, year & Samples & Sample size & MALAT1 pathways in ESCC & (Refs.) \\
\hline Yao, 2019 & $\begin{array}{l}\text { Human ESCC cell lines, normal } \\
\text { human esophageal epithelial cells, } \\
\text { cancer stem cells }\end{array}$ & N/A & $\begin{array}{l}\text { MALAT1 binds to YAP, enhances } \\
\text { YAP protein expression and regulates } \\
\text { the stemness of ESCC cells }\end{array}$ & (13) \\
\hline Chen, 2018 & $\begin{array}{l}\text { Human ESCC cell lines, normal } \\
\text { human esophageal epithelial cells }\end{array}$ & N/A & $\begin{array}{l}\text { MALAT1 affects EMT and } \\
\text { metastasis through the EZH2/Notch1 } \\
\text { signaling pathway }\end{array}$ & $(10)$ \\
\hline Zhang, 2017 & Human ESCC cell lines & N/A & $\begin{array}{l}\text { MALAT1 regulates ZEB1 and ZEB2 } \\
\text { by sponging miR-200a and induces EMT }\end{array}$ & (11) \\
\hline $\mathrm{Li}, 2017$ & Human ESCC cell lines & N/A & $\begin{array}{l}\text { miR-101 and miR-217 act as tumor } \\
\text { suppressor genes by targeting MALAT1 } \\
\text { when delivered via ligand-functionalized } \\
\text { hydroxyl-rich nanovectors }\end{array}$ & (9) \\
\hline $\mathrm{Li}, 2017$ & Mouse ESCC xenografts and cells & N/A & MALAT1 regulates Cks1 expression & (17) \\
\hline Wang, 2016 & $\begin{array}{l}\text { Human ESCC tissues, } \\
\text { human ESCC cell lines, } \\
\text { mouse xenografts }\end{array}$ & $\begin{array}{l}106 \text { patients } \\
\text { (+ adjacent } \\
\text { healthy tissues) }\end{array}$ & $\begin{array}{l}\text { MALAT1 targets } \beta \text {-catenin } \\
\text { via EZH2 }\end{array}$ & (12) \\
\hline Yao, 2016 & $\begin{array}{l}\text { Human ESCC tissues, human } \\
\text { ESCC cell lines }\end{array}$ & $\begin{array}{l}137 \text { patients } \\
(+ \text { adjacent } \\
\text { healthy tissues })\end{array}$ & $\begin{array}{l}\text { MALAT1 contributes to } \mathrm{G} 2 / \mathrm{M} \\
\text { phase arrest, cell apoptosis, } \\
\text { cell migration and invasion }\end{array}$ & (6) \\
\hline $\mathrm{Hu}, 2015$ & $\begin{array}{l}\text { Human ESCC tissues, } \\
\text { human ESCC cell lines }\end{array}$ & $\begin{array}{l}54 \text { patients } \\
\text { (+ adjacent } \\
\text { healthy tissues) }\end{array}$ & $\begin{array}{l}\text { MALAT1 contributes to G2/M } \\
\text { phase arrest and ATM/CHK2 } \\
\text { pathway phosphorylation }\end{array}$ & (7) \\
\hline Wang, 2015 & $\begin{array}{l}\text { Human ESCC tissues, human } \\
\text { ESCC lines, mouse xenografts }\end{array}$ & $\begin{array}{l}42 \text { patients } \\
(+ \text { adjacent } \\
\text { healthy tissues })\end{array}$ & $\begin{array}{l}\text { miR-101 and miR-217 silence } \\
\text { MALAT1, inhibit the migration } \\
\text { and invasion of ESCC cells and } \\
\text { induce G2/M phase arrest }\end{array}$ & (8) \\
\hline
\end{tabular}

MALAT-1, metastasis-associated lung adenocarcinoma transcript 1; ESCC, esophageal squamous cell carcinoma; N/A, not applicable; YAP, yes-associated protein; EMT, epithelial-to-mesenchymal transition; EZH2, enhancer of zeste homolog 2; miR, microRNA; Cks1, cyclin-dependent kinase subunit 1; ATM, ataxia-telangiectasia mutated; CHK2, checkpoint kinase 2.

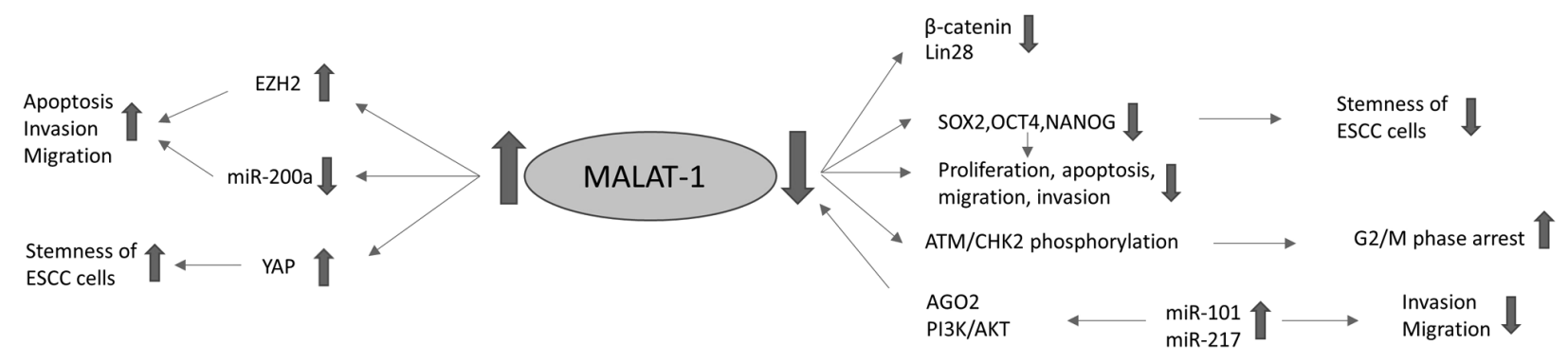

Figure 1. MALAT1 pathways in ESCC. Fine arrows indicate regulatory effects, upward pointing thick arrows indicate overexpression and downward pointing thick arrows indicate downregulation. MALAT-1, metastasis-associated lung adenocarcinoma transcript 1; ESCC, esophageal squamous cell carcinoma; EZH2, enhancer of zeste homolog 2; miR, microRNA; YAP, yes-associated protein; SOX2, sex-determining region Y-box 2; AGO2, argonaute-2.

the overexpression of MALAT1. The authors concluded that MALAT1 is as positive regulator of the radioresistance of ESCC, which may be of great importance for enhancing the radiotherapeutic effect in ESCC.

The main clinical impacts of MALAT1 dysregulation on patients with ESCC are summarized in Table II.

MALAT1 and esophageal adenocarcinoma. At present, to the best of our knowledge, no published literature has investigated in depth the pathways and roles of MALAT1 dysregulation in esophageal adenocarcinomas. However, new possibilities for the diagnosis, prognosis and treatment of patients with esophageal adenocarcinoma may emerge in future studies.

\section{MALAT1 and gastric cancer}

MALAT1 pathways in GC. As research into lncRNAs has increased, new and additional insights into the pathways of 
Table II. Clinical impact of MALAT1 dysregulation on patients with ESCC.

\begin{tabular}{|c|c|c|c|c|}
\hline First author, year & Samples & Sample size & Clinical impact & (Refs.) \\
\hline Yao, 2019 & $\begin{array}{l}\text { Human ESCC cell lines, normal } \\
\text { human esophageal epithelial } \\
\text { cells, cancer stem cells }\end{array}$ & N/A & $\begin{array}{l}\text { Knockdown of MALAT1 enhances } \\
\text { the chemo- and radiosensitivity } \\
\text { of ESCC cells }\end{array}$ & (13) \\
\hline Wang, 2019 & Meta-analysis & 3 studies & $\begin{array}{l}\text { High MALAT1 expression is a } \\
\text { significant independent predictor of } \\
\text { poor overall and disease-free survival }\end{array}$ & (3) \\
\hline Qu, 2019 & Human plasma & $\begin{array}{l}245 \text { ESCC patients } \\
\text { and } 490 \text { controls }\end{array}$ & $\begin{array}{l}\text { MALAT1 polymorphism rs } 3200401 \\
\mathrm{C}>\mathrm{T} \text { increases the risk of ESCC } \\
\text { and polymorphism rs619586 A>G } \\
\text { is protective against ESCC }\end{array}$ & (14) \\
\hline Kang, 2018 & Human ESCC tissues & $\begin{array}{l}100 \text { patients } \\
(+ \text { adjacent } \\
\text { healthy tissues })\end{array}$ & $\begin{array}{l}\text { MALAT1 expression determines } \\
\text { prognosis and is associated with } \\
\text { high TNM stage }\end{array}$ & (15) \\
\hline $\mathrm{Li}, 2017$ & $\begin{array}{l}\text { Mouse ESCC xenografts } \\
\text { and cells }\end{array}$ & N/A & $\begin{array}{l}\text { MALAT1 affects the efficacy of } \\
\text { radiotherapy }\end{array}$ & (17) \\
\hline Huang, 2016 & $\begin{array}{l}\text { Human ESCC tissues, } \\
\text { human EC and esophageal } \\
\text { epithelial cell lines }\end{array}$ & $\begin{array}{l}132 \text { patients, } \\
50 \text { controls }\end{array}$ & $\begin{array}{l}\text { High MALAT1 expression is } \\
\text { associated with lymphatic invasion, } \\
\text { large primary tumor size, distant } \\
\text { metastasis and poor tumor } \\
\text { differentiation }\end{array}$ & (16) \\
\hline $\mathrm{Hu}, 2015$ & $\begin{array}{l}\text { Human ESCC tissues, } \\
\text { human ESCC cell lines }\end{array}$ & $\begin{array}{l}54 \text { patients } \\
\text { (+ adjacent } \\
\text { healthy tissues) }\end{array}$ & $\begin{array}{l}\text { High MALAT1 expression is } \\
\text { associated with lymphatic } \\
\text { invasion and large primary } \\
\text { tumor size }\end{array}$ & (7) \\
\hline
\end{tabular}

MALAT-1, metastasis-associated lung adenocarcinoma transcript 1; ESCC, esophageal squamous cell carcinoma; N/A, not applicable; EC, esophageal carcinoma.

MALAT1 in GC have been gained. EZH2 is overexpressed in various cancer subtypes and contributes to tumorigenesis and progression through the transcriptional silencing of tumor suppressor genes. In GC, the binding of MALAT1 to EZH2 inhibits the expression of the tumor suppressor gene protocadherin 10 and promotes the migration and invasion of GC cells (18). MALAT1 may also promote the proliferation of GC cells via the regulation of splicing factor 2/alternative splicing factor (SF2/ASF) in GC cell lines, MALAT1 induces the upregulation of SF2/ASF in the nucleolus, while the downregulation of MALAT1 or SF2/ASF induces cell cycle arrest at the G0/G1 phase and suppresses cell proliferation. Although the nuclear distribution and expression of SF2/ASF are disturbed when MALAT1 is depleted, the overexpression of SF2/ASF does not attenuate the MALAT1 depletion-induced reduction in cell proliferation (19). MALAT1 knockdown also promotes apoptosis, and suppresses the growth, migration and invasion of GC cells (2).

As aforementioned, MALAT1 functions as a ceRNA, indirectly regulating miRNAs and functionally liberating various RNA transcripts. The expression levels of miR-122 and MALAT1 are negatively associated in gastric cells, while those of MALAT1 and insulin-like growth factor 1 receptor(IGF-1R), an miR-122 target, are positively associated. Furthermore, the overexpression of miR-122 inhibits MALAT1 expression, while the inhibition of miR-122 upregulates MALAT1 overexpression. This suggests that the miR-122/IGF-1R axis may modulate the expression of MALAT1 in GC cells (20). Zhang et al (21) demonstrated that MALAT1 directly targets miR-202 and the downregulation of MALAT1 significantly decreases the expression of Gli2 via the negative regulation of miR-202. They also revealed that the knockdown of MALAT1 in GC cells inhibits cell proliferation, reduces the number of cells in the S-phase, and induces apoptosis via the negative regulation of miR-202. Li et al (22) identified a negative association between MALAT1 and miR-1297, and revealed that MALAT1 promotes high mobility group protein B2 (HMGB2) through the negative regulation of miR-1297. Their results indicated that the MALAT1/miR-1297/HMGB2 axis is important for the regulation of GC tumorigenesis and progression, and may serve as a therapeutic target for GC. In a study conducted by Li et al (23), transfected cells with overexpressed or silenced MALAT1 were prepared, and their proliferation and apoptosis, as well as the tumor volume and weight when injected into mice were evaluated. The overexpression of MALAT1 promoted proliferation and inhibited apoptosis via the upregulation of miR-22-3p and downregulation of ErbB3, while the knockdown of MALAT1 inhibited GC tumor growth in mice. Zhang et al (24) demonstrated that the sponging of miR-22-3p by MALAT1 modulated the oxaliplatin resistance of GC xenografts. The knockdown of MALAT1 or overexpression of miR-22-3p inhibited the survival, proliferation and 
drug resistance of GCs subjected to oxaliplatin treatment, and those effects were attenuated by the inhibition of miR-22-3p.

The interleukin-21 receptor (IL-21R) contributes to the progression of GC, and its knockdown suppresses the invasion and proliferation of GC cells. The expression of IL-21R is negatively associated with that of miR-125a-3p, a tumor suppressive miRNA, in GC, and MALAT1 is able to sponge miR-125a-3p. It appears that the upregulation of MALAT1 dysregulates the MALAT1/miR-125a-3p axis, which leads to the activation of IL-21R and its oncogenic role in GC (25).

The levels of MALAT1 are elevated in the serum of patients with gastric adenocarcinoma and in gastric adenocarcinoma cell lines, and the knockdown of MALAT1 inhibits the proliferation of gastric adenocarcinoma cells and promotes their apoptosis. MALAT1 directly targets and decreases the expression of miR-181a-5p, which upregulates the expression of AKT3 and promotes tumor growth (26).

Activation of the PI3K/AKT pathway increases the migration, proliferation and invasion of GC cells. MALAT1 increases the phosphorylation levels of PI3K and AKT, which may promote the progression of GC (27). The cisplatin resistance of GC has also been attributed to the regulatory effect of MALAT1 on the PI3K/AKT pathway (4).

The expression of miR-183 is downregulated in GC cells, and the knockdown of miR-183 has been shown to increase the viability and reduce the apoptosis of $\mathrm{GC}$ cells by modulating the activation of cell autophagy. The underlying mechanisms have been suggested to involve the MALAT1/miR-183/sirtuin 1 axis and PI3K/AKT/mTOR pathway, and miR-183 has been proposed as a therapeutic target for GC due to its dysregulating effect on those pathways (28). Elevated levels of MALAT1 are associated with increased levels of microtubule-associated protein 1 light chain $3 \beta$ (LC3B), an autophagy marker, and antigen Ki67, a proliferation marker. miR-204 represses the autophagy of $\mathrm{GC}$ cells through the downregulation of LC3B and transient receptor potential melastatin 3 expression, while MALAT1 inhibits the expression of miR-204 and thereby activates autophagy (5). Studies of human gastric tumors have been conducted to elucidate the functional role of MALAT1 in autophagy-associated chemoresistance. Chemoresistant GC cells exhibit elevated levels of MALAT1 and increased autophagy. The chemo-induced autophagy of GC cells is promoted by MALAT1 and the knockdown of MALAT1 sensitizes GC cells to chemotherapeutics. A proposed mechanism is that MALAT1 acts as a ceRNA for miR-23b-3p and attenuates the inhibitory effect of miR-23b-3p on the autophagy regulator ATG12, leading to elevated ATG12 levels, chemo-induced autophagy and chemoresistance in GC cells (29). Also, MALAT1 has been shown to potentiate autophagy-associated cisplatin resistance via suppression of the miR-30b/ATG5 axis and the facilitation of autophagy, indicating that MALAT1 may be targeted to overcome chemoresistance in GC. MALAT1 directly binds to miR-30b and inhibits its expression, and the upregulation of miR-30b attenuates MALAT1-induced cisplatin resistance via the suppression of autophagy (30). Another study confirmed that GC cells presented increased autophagy and high expression levels of MALAT1, and demonstrated that propofol induced apoptosis and reduced autophagic activity through the downregulation of MALAT1. The knockdown of MALAT1 inhibited chemo-induced autophagy, and MALAT1 overexpression promoted autophagy by directly binding with miR-30e and thereby regulating ATG5 expression. Furthermore, in a GC xenograft model, mice treated with both propofol and cisplatin exhibited a significant reduction in tumor size and weight, and the knockdown of MALAT1 enhanced these effects (31).

Up-frameshift suppressor 1 homolog (UPF1) has been shown to be a modulator of MALAT1, and the UPF1/MALAT1 pathway may be a potential therapeutic target for GC. The overexpression of UPF1 inhibits proliferation, cell cycle, migration and invasion, and promotes cell apoptosis in GC cells, and the overexpression of MALAT1 attenuates the UPF1-mediated inhibition of GC progression. The overexpression of UPF1 is indicated to downregulate MALAT1 by increasing the efficiency of nonsense-mediated mRNA decay (32).

MALAT1 has been demonstrated to promote tumorigenesis and metastasis in GC by facilitating vasculogenic mimicry and angiogenesis via the vascular endothelial (VE)-cadherin/ $\beta$-catenin complex and ERK/matrix metalloproteinase (MMP) and focal adhesion kinase (FAK)/paxillin signaling pathways. The mechanism of MALAT1 involves the modulation of VE-cadherin, $\beta$-catenin, MMP2, MMP9 and membrane type 1-MMP expression, and phosphorylated (p)-ERK, p-FAK and p-paxillin levels, which are classical markers of vasculogenic mimicry and angiogenesis and components of several associated signaling pathways (33).

In a study of GC, MALAT1 silencing promoted a reduction in GC cell invasion and migration, and those effects were reversed by transfection with epidermal growth factor-like domain-containing protein 7 (EGFL7). Furthermore, in a GC xenotransplant mouse model, the downregulation of MALAT1 inhibited tumor metastasis. MALAT1 was demonstrated to regulate EGFL7 expression by altering the $\mathrm{H} 3$ histone acetylation level in the EGFL7 promoter (34).

MALAT1 promotes invasion and migration in GC via EMT, with reduced expression of snail and N-cadherin, and increased expression of E-cadherin. Transfection with MALAT1 siRNA significantly decreases the expression of Wnt $/ \beta$-catenin-associated genes (35). Also, the suppression of MALAT-1 expression decreases the expression of the EMT-associated marker vimentin and increases the expression of E-cadherin (36). Yang et al (37) investigated the anticancer effects of resveratrol, a traditional Chinese medicine, and found that they proceeded via the inhibition of MALAT1 expression. EMT was suppressed by resveratrol, and the knockdown of MALAT1 expression suppressed the viability, migration, invasion and EMT of EC cells (37).

The MALAT1/SOX2 axis has been reported to induce the stemness of GC cells. The knockdown of MALAT1 attenuates the stemness of non-adherent GC cell spheroids and, conversely, the overexpression of MALAT1 increases the stemness of adherent GC cells. The expression of MALAT1 and SOX2 is positively correlated. MALAT1 directly binds to SOX2 mRNA and increases its expression. The promoting effect of MALAT1 overexpression on the stemness of GC cells is attenuated by the knockdown of SOX2 (38).

MALAT1 pathways in GC are summarized in Table III and presented in Fig. 2 


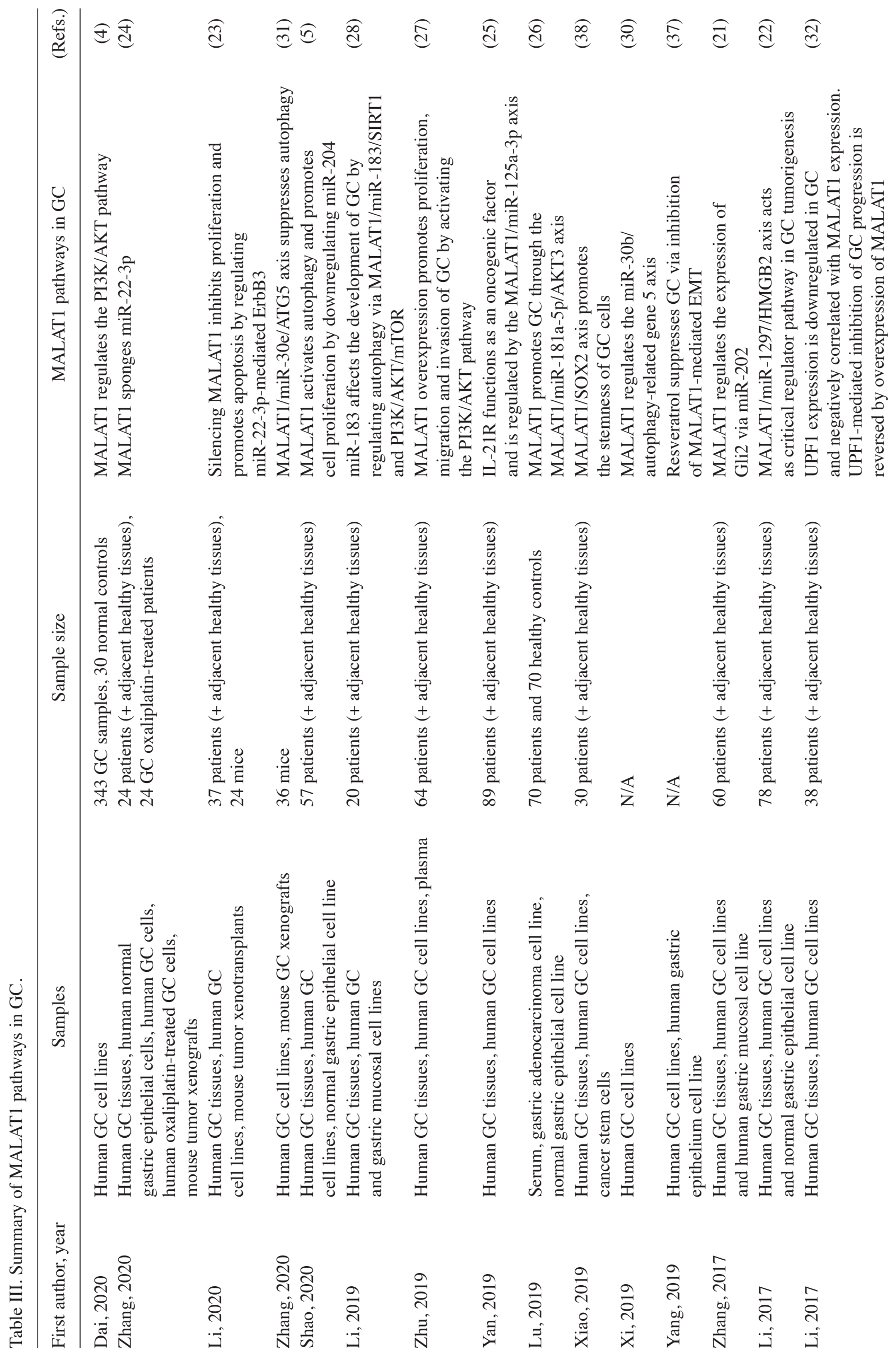




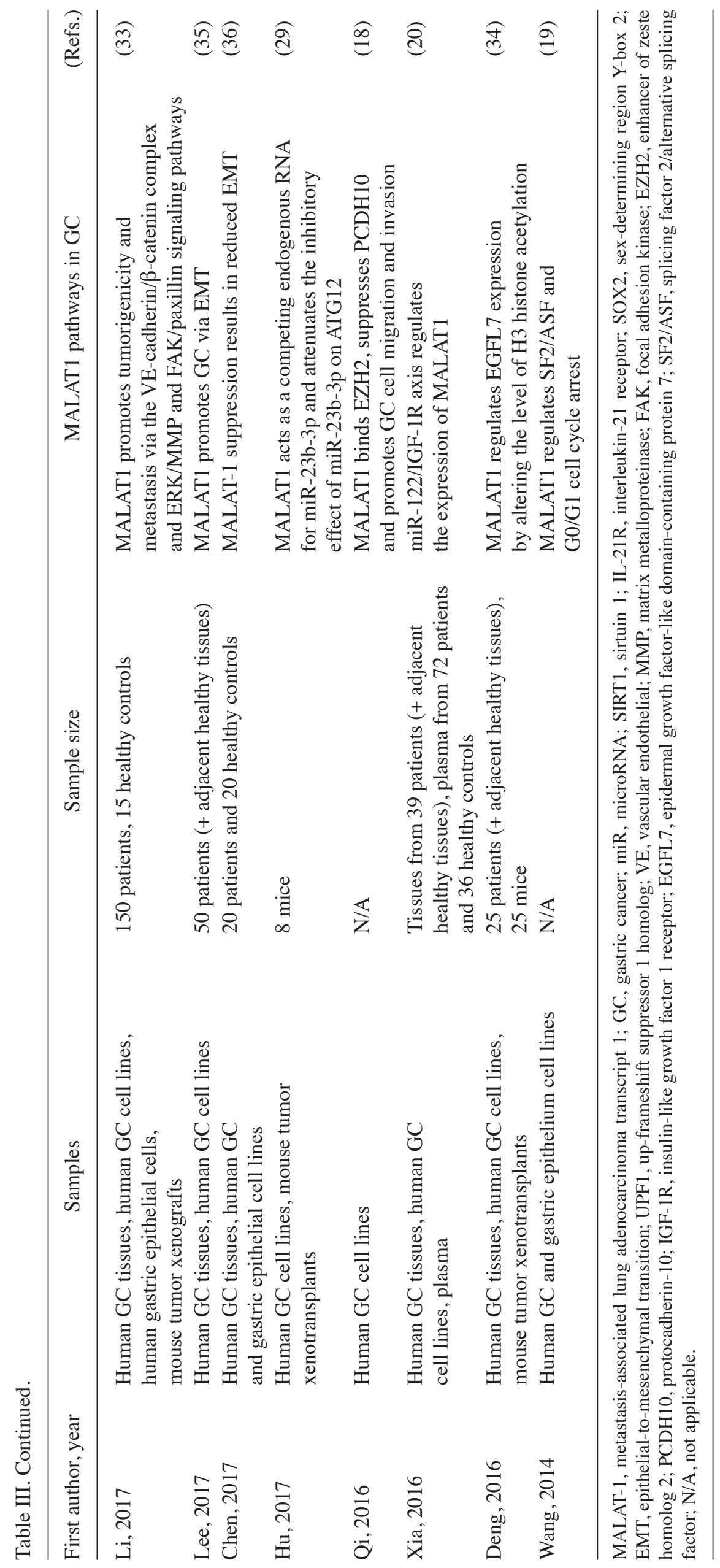




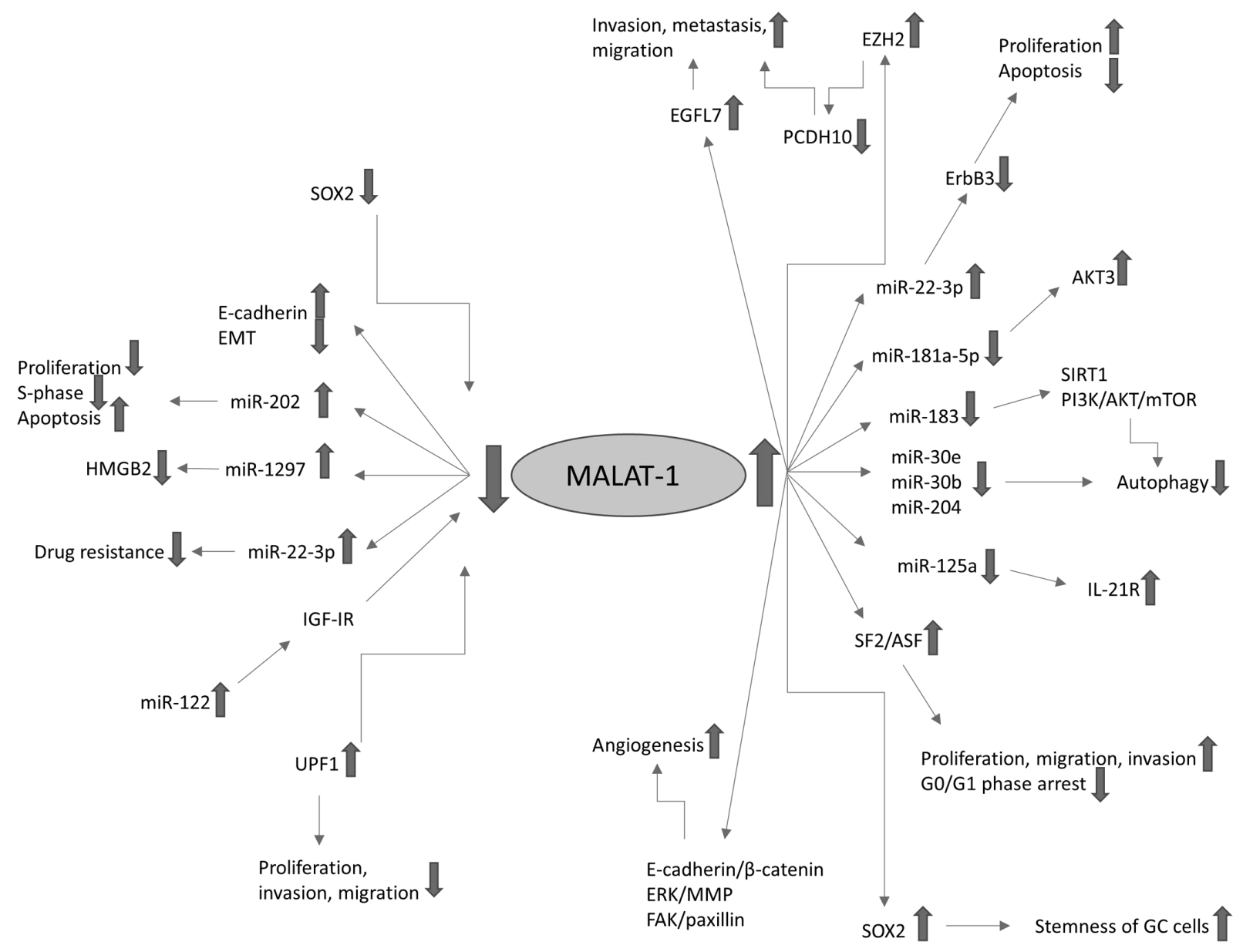

Figure 2. MALAT1 pathways in GC. Fine arrows indicate regulatory effects, upward pointing thick arrows represent overexpression and downward pointing thick arrows indicate downregulation. MALAT-1, metastasis-associated lung adenocarcinoma transcript 1; GC, gastric cancer; miR, microRNA; EMT, epithelial-to-mesenchymal transition; SOX2, sex-determining region Y-box 2; HMGB2, high mobility group protein B2; IGF-1R, insulin-like growth factor 1 receptor; UPF1, up-frameshift suppressor 1 homolog; MMP, matrix metalloproteinase; FAK, focal adhesion kinase; EZH2, enhancer of zeste homolog 2; EGFL7, epidermal growth factor-like domain-containing protein 7; PCDH10, protocadherin-10; SIRT1, sirtuin 1; IL-21R, interleukin-21 receptor; SF2/ASF, splicing factor $2 /$ alternative splicing factor.

Impact of MALATl expression on patients with GC. In patients with GC, it has been observed that MALAT1 is upregulated. In particular, MALAT1 is overexpressed in the serum of patients with gastric adenocarcinoma and GC tissues, as well as in GC cell lines $(23,26)$.

It is known that lifestyle choices such as vegetarianism, smoking and drinking may affect the expression of lncRNAs; for example, the expression of the IncRNA SCAL1 may be induced by smoking (27). This may impact the diagnostic and prognostic utility of a particular lncRNA. To the best of our knowledge, the only study that has investigated the effects of patients' lifestyle choices on the expression of MALAT1 in GC is a study by Zhu et al (27), which recorded MALAT1 expression levels in patients with different lifestyles. The study found that MALAT1 expression levels in the plasma and tumor tissues of patients GC are not significantly associated with sex, age, smoking, drinking or vegetarianism. These results indicate that plasma MALAT1 levels may be used for the accurate diagnosis of GC in patients with different lifestyles $(27,35)$.

Elevated MALAT1 expression is associated with a larger tumor size, lymph node metastasis and higher TNM stage $(21,22)$. Patients with elevated levels of MALAT1 expression are likely to have a greater depth of tumor invasion and more advanced TNM stage compared with patients who have lower levels of MALAT1. MALAT1 expression is also higher in patients with GC with poorly differentiated histology or signet ring cell carcinoma than in those with well-to-moderately differentiated adenocarcinoma (35). In addition, the upregulation of MALAT1 promotes the metastasis and peritoneal dissemination of GC, while the downregulation of MALAT1 inhibits tumor metastasis $(20,33,34,36,39)$. Therefore, the expression level of MALAT1 is a potential biomarker for the distant metastasis of GC. Okugawa et al (39) and Wang et al (3) did not observe that patients with high MALAT1 expression have a poorer prognosis than patients with low MALAT1 expression, whereas Xia et al (20) and Li et al (23) found that high levels of plasma MALAT1 were independently associated with a worse prognosis for patients with GC. Li et al (22) also reported that higher MALAT1 expression predicted a shorter survival and poor prognosis, and Dai et al (4) proposed that the upregulation of MALAT1 in GC tissues is an independent risk factor for OS among patients with GC. Moreover, Qi et al (18) found that the high expression of MALAT1 may be significantly associated with poor OS among patients with 


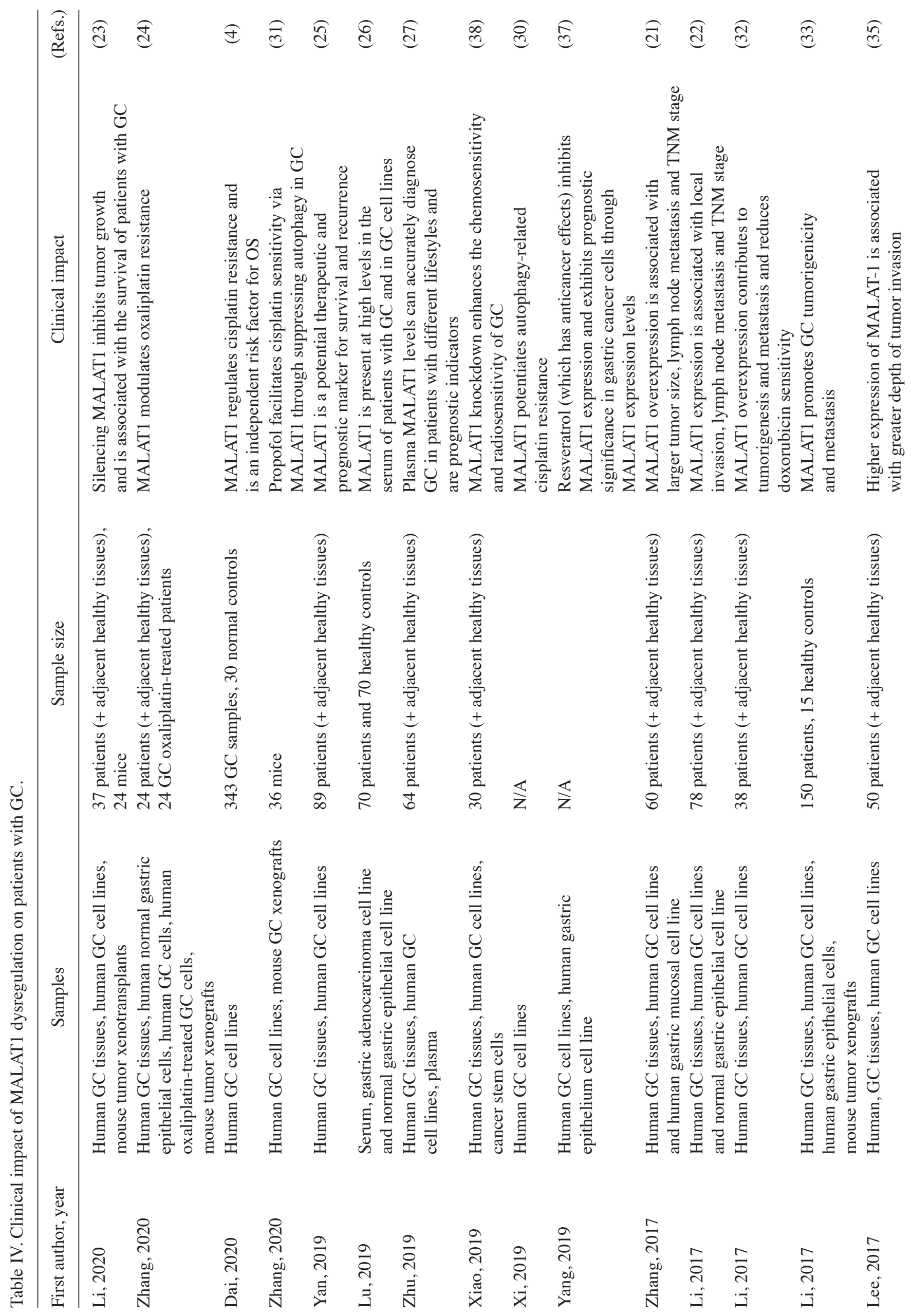


stage III or IV GC, but not those with stage I and II GC. Finally, Yan et al (25) suggested that MALAT1 contributes to gastric tumorigenesis and is a risk factor for survival and recurrence since patients with GC with high MALAT1 expression exhibit a lower survival rate and higher probability of recurrence compared with those with low MALAT1 expression. These data suggest that MALAT1 may serve as a diagnostic and prognostic indicator in $\mathrm{GC}$, and is able to provide an indication of the severity of the disease.

Chemoresistance and radioresistance are major obstacles to the successful treatment of GC, and MALAT1 may serve as a potential therapeutic marker. The overexpression of MALAT1 reduces chemotherapeutical sensitivity, while the knockdown of MALAT1 improves the chemosensitivity of GC $(2,29,32)$. The overexpression of MALAT1 reduces doxorubicin sensitivity and potentiates cisplatin resistance in GC, while the knockdown of MALAT1 inhibits oxaliplatin resistance $(4,30,24,32)$. In addition, the knockdown of MALAT1 enhances the radiosensitivity of GC cells while MALAT1 overexpression attenuates it (38).

The main clinical impacts of MALAT1 dysregulation in patients with GC are summarized in Table IV.

\section{Conclusions}

The identification of biomarkers and use of molecular targeting has expanded the field of cancer therapeutics. The research and identification of IncRNAs and their interactions with oncogenic pathways offer crucial information to the clinical aspects of cancer management. Research elucidating the pathways of MALAT1 in esophagogastric malignancies is suggesting new possibilities for the diagnosis, prognosis and therapy of GC and EC. MALAT1 appears to be a promising diagnostic, prognostic and therapeutic biomarker in esophagogastric malignancies. Ongoing research into its interaction with different oncogenic pathways may provide new avenues for EC and GC therapy.

\section{Acknowledgements}

Not applicable.

\section{Funding}

No funding was received.

\section{Availability of data and materials}

The datasets used and/or analyzed during the current study are available from the corresponding author on reasonable request.

\section{Authors' contributions}

AS contributed to study conception and design, the acquisition of data and drafting the manuscript. DM contributed to drafting the manuscript and the analysis and interpretation of data. GSK, SS and IK contributed to the analysis and interpretation of data and drafting the manuscript. MG contributed to the analysis and interpretation of data, and the drafting and 
critical revision of the manuscript. DS contributed to study conception and design, the acquisition of data, and the drafting and critical revision of the manuscript. All authors read and approved the final manuscript.

\section{Ethics approval and consent to participate}

Not applicable.

\section{Patient consent for publication}

Not applicable.

\section{Competing interests}

The authors declare that they have no competing interests.

\section{References}

1. Torre LA, Siegel RL, Ward EM and Jemal A: Global Lnds - an update. Cancer Epidemiol Biomarkers Prev 25: 16-27, 2016.

2. Farooqi AA, Legaki E, Gazouli M, Rinaldi S and Berardi R: Withdrawal Notice: MALAT1 as a versatile regulator of cancer: overview of the updates from predatory role as competitive endogenous RNA to mechanistic in-sights. Curr Cancer Drug Targets: Jul 30, 2020 (Epub ahead of print).

3. Wang C, Zhang Q, Hu Y, Zhu J and Yang J: Emerging role of long non-coding RNA MALAT1 in predicting clinical outcomes of patients with digestive system malignancies: A meta-analysis. Oncol Lett 17: 2159-2170, 2019.

4. Dai Q, Zhang T and Li C: LncRNA MALAT1 regulates the cell proliferation and cisplatin resistance in gastric cancer via PI3K/AKT pathway. Cancer Manag Res 12: 1929-1939, 2020.

5. Shao G, Zhao Z, Zhao W, Hu G, Zhang L, Li W, Xing C and Zhang X: Long non-coding RNA MALAT1 activates autophagy and promotes cell proliferation by downregulating microRNA-204 expression in gastric cancer. Oncol Lett 19: $805-812,2020$

6. Yao W, Bai Y, Li Y, Guo L, Zeng P, Wang Y, Qi B, Liu S, Qin X, Li Y, et al: Upregulation of MALAT-1 and its association with survival rate and the effect on cell cycle and migration in patients with esophageal squamous cell carcinoma. Tumour Biol 37 4305-4312, 2016.

7. Hu L, Wu Y, Tan D, Meng H, Wang K, Bai Y and Yang K: Up-regulation of long noncoding RNA MALAT1 contributes to proliferation and metastasis in esophageal squamous cell carcinoma. J Exp Clin Cancer Res 34: 7, 2015.

8. Wang X, Li M, Wang Z, Han S, Tang X, Ge Y, Zhou L, Zhou C, Yuan $\mathrm{Q}$ and Yang M: Silencing of long noncoding RNA MALAT1 by miR-101 and miR-217 inhibits proliferation, migration, and invasion of esophageal squamous cell carcinoma cells. J Biol Chem 290: 3925-3935, 2015.

9. Li RQ, Ren Y, Liu W, Pan W, Xu FJ and Yang M: MicroRNA-mediated silence of onco-lncRNA MALAT1 in different ESCC cells via ligand-functionalized hydroxyl-rich nanovectors. Nanoscale 9: 2521-2530, 2017.

10. Chen M, Xia Z, Chen C, Hu W and Yuan Y: LncRNA MALAT1 promotes epithelial-to-mesenchymal transition of esophageal cancer through Ezh2-Notch1 signaling pathway. Anticancer Drugs 29: 767-773, 2018

11. Zhang QQ, Cui YH, Wang Y, Kou WZ, Cao F, Cao XJ, Miao ZH and Kang XH: Mechanism of long non-coding RNA-metastasis associated lung adenocarcinoma transcript 1 induced invasion and metastasis of esophageal cancer cell EC-109. Zhonghua Zhong Liu Za Zhi 39: 405-411, 2017 (In Chinese).

12. Wang W, Zhu Y, Li S, Chen X, Jiang G, Shen Z, Qiao Y, Wang L, Zheng $\mathrm{P}$ and Zhang Y: Long noncoding RNA MALAT1 promotes malignant development of esophageal squamous cell carcinoma by targeting $\beta$-catenin via Ezh2. Oncotarget 7: 25668-25682, 2016.

13. Yao Q, Yang J, Liu T, Zhang J and Zheng Y: Long noncoding RNA MALAT1 promotes the stemness of esophageal squamous cell carcinoma by enhancing YAP transcriptional activity. FEBS Open Bio 9: 1392-1402, 2019.
14. Qu Y, Shao N, Yang W, Wang J and Cheng Y: Association of polymorphisms in MALAT1 with the risk of esophageal squamous cell carcinoma in a Chinese population. Onco Targets Ther 12: 2495-2503, 2019

15. Kang K, Huang YH, Li HP and Guo SM: Expression of UCA1 and MALAT1 long-chain non-coding RNAs in esophageal squamous cell carcinoma tissues is predictive of patient prognosis. Arch Med Sci 14: 752-759, 2018.

16. Huang C, Yu Z, Yang H and Lin Y: Increased MALAT1 expression predicts poor prognosis in esophageal cancer patients. Biomed Pharmacother 83: 8-13, 2016.

17. Li Z,Zhou Y,Tu B, Bu Y, Liu A and Kong J: Long noncoding RNA MALAT1 affects the efficacy of radiotherapy for esophageal squamous cell carcinoma by regulating Cks1 expression. J Oral Pathol Med 46: 583-590, 2017.

18. Qi Y, Ooi HS, Wu J, Chen J, Zhang X, Tan S, Yu Q, Li YY, Kang Y, Li H, et al: MALAT1 long ncRNA promotes gastric cancer metastasis by suppressing PCDH10. Oncotarget 7: 12693-12703, 2016.

19. Wang J, Su L, Chen X, Li P, Cai Q, Yu B, Liu B, Wu W and Zhu Z: MALAT1 promotes cell proliferation in gastric cancer by recruiting SF2/ASF. Biomed Pharmacother 68: 557-564, 2014.

20. Xia H, Chen Q, Chen Y, Ge X, Leng W, Tang Q, Ren M, Chen L, Yuan D, Zhang Y, et al: The IncRNA MALAT1 is a novel biomarker for gastric cancer metastasis. Oncotarget 7: 56209-56218, 2016.

21. Zhang Y, Chen Z, Li MJ, Guo HY and Jing NC: Long non-coding RNA metastasis-associated lung adenocarcinoma transcript 1 regulates the expression of Gli2 by miR-202 to strengthen gastric cancer progression. Biomed Pharmacother 85: 264-271, 2017.

22. Li J, Gao J, Tian W, Li Y and Zhang J: Long non-coding RNA MALAT1 drives gastric cancer progression by regulating HMGB2 modulating the miR-1297. Cancer Cell Int 17: 44, 2017.

23. Li X, Zhao J, Zhang $\mathrm{H}$ and Cai J: Silencing of IncRNA metastasis-associated lung adenocarcinoma transcript 1 inhibits the proliferation and promotes the apoptosis of gastric cancer cells through regulating microRNA-22-3p-mediated ErbB3. Onco Targets Ther 13: 559-571, 2020.

24. Zhang Z, Li M and Zhang Z: IncRNA MALAT1 modulates oxaliplatin resistance of gastric cancer via sponging miR-22-3p. Onco Targets Ther 13: 1343-1354, 2020.

25. Yan L, Zhang J, Guo D, Ma J, Shui SF and Han XW: IL-21R functions as an oncogenic factor and is regulated by the lncRNA MALAT1/miR-125a-3p axis in gastric cancer. Int J Oncol 54: 7-16, 2019.

26. Lu Z, Luo T, Pang T, Du Z, Yin X, Cui H, Fang G and Xue X: MALAT1 promotes gastric adenocarcinoma through the MALAT1/miR-181a-5p/AKT3 axis. Open Biol 9: 190095, 2019.

27. Zhu K, Ren Q and Zhao Y: lncRNA MALAT1 overexpression promotes proliferation, migration and invasion of gastric cancer by activating the PI3K/AKT pathway. Oncol Lett 17: 5335-5342, 2019.

28. Li H,He C, Wang X, Wang H, Nan G and Fang L: MicroRNA-183 affects the development of gastric cancer by regulating autophagy via MALAT1-miR-183-SIRT1 axis and PI3K/AKT/mTOR signals. Artif Cells Nanomed Biotechnol 47: 3163-3171, 2019.

29. Hu YR, Yu YC, You S, Li K, Tong X, Chen S, Chen E, Lin XZ and Chen Y: Long noncoding RNA MALAT1 regulates autophagy associated chemoresistance via miR-23b-3p sequestration in gastric cancer. Mol Cancer 16: 174, 2017.

30. Xi Z, Si J and Nan J: LncRNA MALAT1 potentiates autophagy-associated cisplatin resistance by regulating the microRNA-30b/autophagy-related gene 5 axis in gastric cancer. Int J Oncol 54: 239-248, 2019.

31. Zhang YF, Li CS, Zhou Y and Lu XH: Propofol facilitates cisplatin sensitivity via lncRNA MALAT1/miR-30e/ATG5 axis through suppressing autophagy in gastric cancer. Life Sci 244: 117280,2020

32. Li L, Geng Y, Feng R, Zhu Q, Miao B, Cao J and Fei S: The human RNA surveillance factor UPF1 modulates gastric cancer progression by targeting long non-coding RNA MALAT1. Cell Physiol Biochem 42: 2194-2206, 2017.

33. Li Y, Wu Z, Yuan J, Sun L, Lin L, Huang N, Bin J, Liao Y and Liao W: Long non-coding RNA MALAT1 promotes gastric cancer tumorigenicity and metastasis by regulating vasculogenic mimicry and angiogenesis. Cancer Lett 395: 31-44, 2017.

34. Deng QJ, Xie LQ and Li H: Overexpressed MALAT1 promotes invasion and metastasis of gastric cancer cells via increasing EGFL7 expression. Life Sci 157: 38-44, 2016 
35. Lee NK, Lee JH, Ivan C, Ling H, Zhang X, Park CH, Calin GA and Lee SK: MALAT1 promoted invasiveness of gastric adenocarcinoma. BMC Cancer 17: 46, 2017.

36. Chen D, Liu L, Wang K, Yu H, Wang Y, Liu J, Guo Y and Zhang H: The role of MALAT-1 in the invasion and metastasis of gastric cancer. Scand J Gastroenterol 52: 790-796, 2017.

37. Yang Z, Xie Q, Chen Z, Ni H, Xia L, Zhao Q, Chen Z and Chen P: Resveratrol suppresses the invasion and migration of human gastric cancer cells via inhibition of MALAT1-mediated epithelial-to-mesenchymal transition. Exp Ther Med 17: $1569-1578,2019$.
38. Xiao Y, Pan J, Geng Q and Wang G: LncRNA MALAT1 increases the stemness of gastric cancer cells via enhancing SOX2 mRNA stability. FEBS Open Bio 9: 1212-1222, 2019.

39. Okugawa Y, Toiyama Y, Hur K, Toden S, Saigusa S, Tanaka K, Inoue Y, Mohri Y, Kusunoki M, Boland CR, et al: Metastasis-associated long non-coding RNA drives gastric cancer development and promotes peritoneal metastasis. Carcinogenesis 35: 2731-2739, 2014. 